\title{
BEOBACHTUNGEN \\ AM AMAZONAS UND RIO NEGRO
}

\author{
Rudolf Streiff-Becker
}

Mit 6 Abbildungen

Im Jahre 1957 bereiste der Brasilianer Rubens Rodrigues Dos SAntos zu Studienzwecken den mittleren Teil des Amazonasgebietes. Er veröffentlichte seine Ergebnisse in der Zeitung «O Estado de S. Paulo» im Frühjahr 1958, unter dem Titel «Viagem ao rio da sombra e do Silencio». Im Glauben, daß der Inhalt dieses Reiseberichtes Geographen und Ethnographen interessieren werde, bringt der Autor dieses Beitrages einen Auszug in freier Übersetzung nach dem portugiesischen Originaltext.

Die großen Ströme im Gebiet der Amazonasniederung fließen nicht zwischen mehr oder weniger parallelen Ufern dahin, sondern zweigen im flachen Gelände seitliche Nebenrinnen ab, die sich nach längerem oder kürzeren Lauf mit dem Hauptstrom wieder vereinigen, unzählige sandige, oft mit Regenwald bestandene Inseln bildend. Die breiten Flußabzweigungen nennt der Indianer «Paranà» (großes Wasser), die kleineren «Igarapé» (Weg für Canoa). Beide sind für den Eingeborenen von größter Bedeutung. Wie der Bewohner trockener Landstriche Pisten und Straßen benötigt, so sind dem Bewohner des Amazonasgebietes die Igarapés die Verkehrswege, die zudem keinen Unterhalt erheischen, ihm Wasser zum Trinken und Waschen zur Verfügung halten und ihm reichlich Nahrung liefern in Fischen, Schildkröten, Krustentieren und Krokodilen. Die Häute der letzteren kann er zu guten Preisen verkaufen. Mit seiner Canoa kann er auch in sonst schwer zugängliche Gebiete des Regenwaldes dringen, wo er jagdbare Tiere und die für ihn so wichtigen Gummibäume Maniçoba oder Seringueira (Hevea brasiliensis) findet. Der von diesen Bäumen gewonnene Saft, genannt Latex, verarbeitet er zu Rohkautschuk, und der Verkauf dieses so begehrten Produktes bringt ihm Bargeld zum Kauf fremder Waren. Beim Durchfahren des ruhigen Wassers im Igarapé, im Schatten der breiten Baumkronen bedrohen den Schiffer außerdem weniger Gefahren als im breiten offenen Wasser der Hauptströme, wo bei Gewitterstürmen hoher Wellengang vorkommen kann.

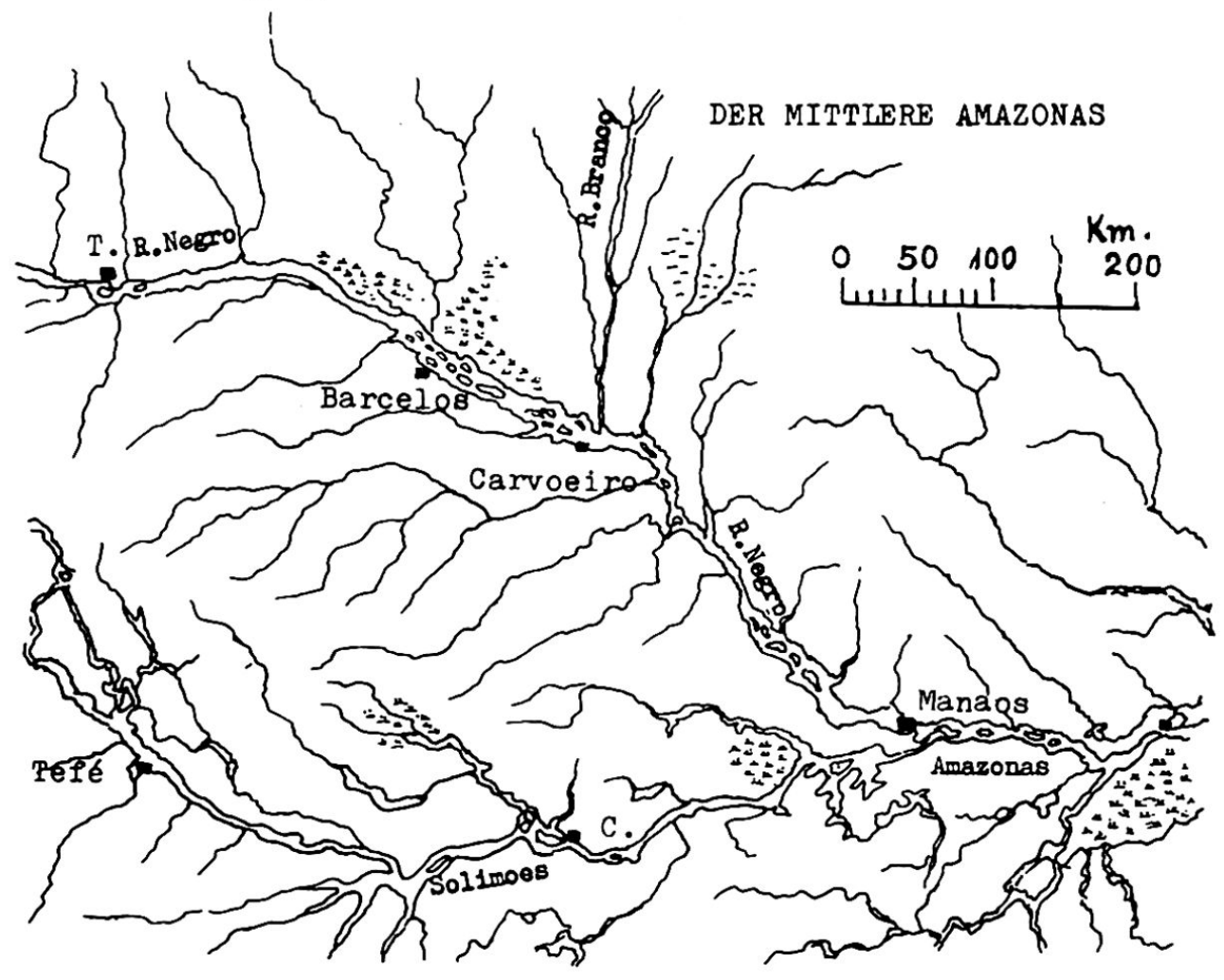


Die Farbe des Wassers gibt den Strömen vielfach deren Namen. Das Wasser des Rio Branco ist meistens klardurchsichtig, entsprechend dem Ursprung aus Gebieten kristallinen Gesteins; das Wasser des Rio Negro dagegen ist fast immer sepiafarben, weil an seinen Ufern dunkle Tone anstehen, während der hellockerfarbene Solimoes von seinen Ufern Verwitterungsprodukte gelblicher Gesteine wegspült. Die Farbe wird aber auch durch Pflanzensäfte und namentlich durch Algen beeinflußt. Wegen dem geringen Gefälle ist die Strömung sehr langsam, so daß nach dem Zusammenfluß der Ströme deren Wasser noch auf langen Strecken ihren Farben entsprechend deutlich getrennt nebeneinander dahin fließen. Die Fahrt durch die Igarapés fesselt durch den Anblick des üppigen Regenwaldes, während diejenige auf dem offenen großen Strom wegen der Weite sehr eintönig ist. Man sieht da nur Himmel und Wasser, am fernen Horizont getrennt durch ein schmales blaugraues Band.

Manaos, die Hauptstadt des brasilianischen Staates Amazonas, liegt rund $1200 \mathrm{~km}$ vom atlantischen Ozean entfernt, nur $26 \mathrm{~m}$. ü. M., bei $60^{\circ}$ w. L. und $3^{\circ} \mathrm{s}$. Br., am Nordufer des Rio Negro, nahe bei dessen Mündung in den größeren Solimoes (Amazonas). Die Eingeborenen nennen den vereinigten Strom bis zu dessen Mündung ins Meer «Amazonas». Manaos ist eine Stadt, deren Entwicklungsgeschichte beinahe dramatische Züge aufweist. Noch zu Anfang dieses Jahrhunderts war es eine kleine Landstadt, fern von den Kulturzentren des Südostens, mit kaum 10000 Einwohnern. Sie stieg dann in fabelhaft kurzer Zeit zur weltbekannten Großstadt mit rund 250000 Einwohnern empor, um bald darauf wieder zur Kleinstadt zurück zu sinken, mit deutlichen Anzeichen von Dekadenz. Die während der Hochkonjunktur errichteten öffentlichen und Privatgebäude stehen heute vernachläßigt da. Es war der Kautschuk, der das Schicksal von Manaos bestimmte. Als zu Anfang dieses Jahrhunderts das Automobil in Gebrauch kam, wurde der Kautschuk zur Fabrikation det Pneus in steigendem Maße gesucht. Sein Handelswert stieg gewaltig und Manaos erlebte seine Blütezeit. Brasilien erließ ein strenges Verbot der Ausfuhr von Samen oder Stecklingen des Gummibaumes; vergebens. Es gelang dem Schmuggel, Stecklinge nach Ostindien zu bringen, wo bald Großpflanzungen entstanden. Bald machte dieser Plantagenkautschuk, später dazu der synthetische dem Wildkautschuk Brasiliens schwere Konkurrenz. Manaos fiel infolgedessen wirtschaftlich stark zurück. Zur Zeit der Hochkonjunktur gründeten Amerikaner «The Amazonas River Steam Navigation Company», welche mit Motorschiffen auch die wichtigeren Nebenflüsse bis an die Landesgrenzen befuhr. Diese Gesellschaft wurde später verstaatlicht unter dem Namen SNAPP (Abkürzung für «Serviçio de Navegaçao da Amazonia e Administraçao do Porto do Parà). Das Volk gibt den Schiffen eigene, oft witzige, Namen. So nennt es Schiffe, die neben Waren auch Passagiere und Begleittiere befördern «Gaiolas» (Vogelkäfig), weil sie solchen ähnlich sehen durch die vielen Gitter und Stangen, welche dem Verdeck aufgesetzt sind. Größere, mit mehr Luxus ausgestattete Schiffe heißen «Vaticanos», solche mit sehr flachem Kiel zur Benützung bei Niederwasser sind

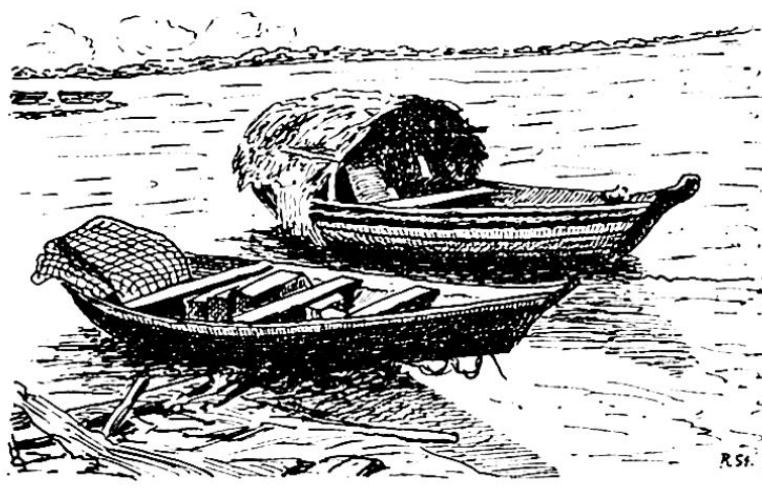

Canoas mit Schutzdach (Igarité)

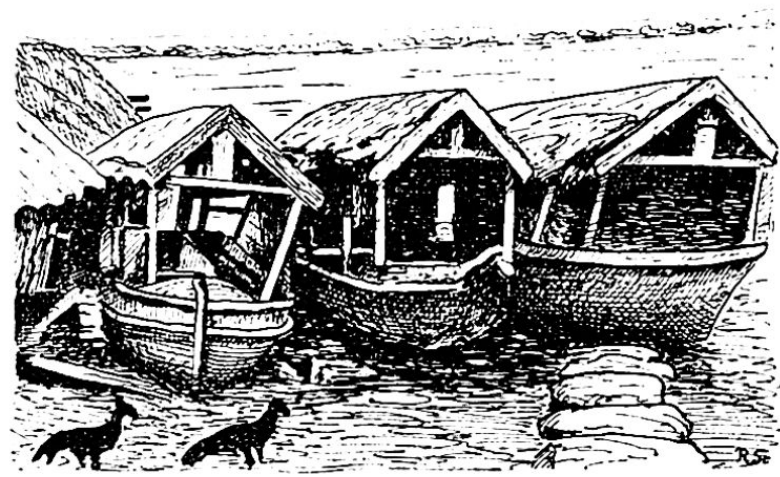

Canoas mit Hüttenaufbau (Batalaõs) 
«Chatas» (Die Flachen). Schließlich nennt man Schiffe, die als ambulante Verkaufsläden von Ort zu Ort gefahren werden «Regatoes» (Krämer). Mit der Verstaatlichung der Schiffahrt und wohl auch wegen dem Rückgang der Konjunktur geriet sie in Verfall. Die Bundesregierung mußte eingreifen. Seit 1955 versehen neue gute Dampfer den Dienst auf den Hauptströmen. In den Außenquartieren der Stadt, wo die Caboclos wohnen, sind verschiedene Schiffstypen im Gebrauch. Die einfache Ca-

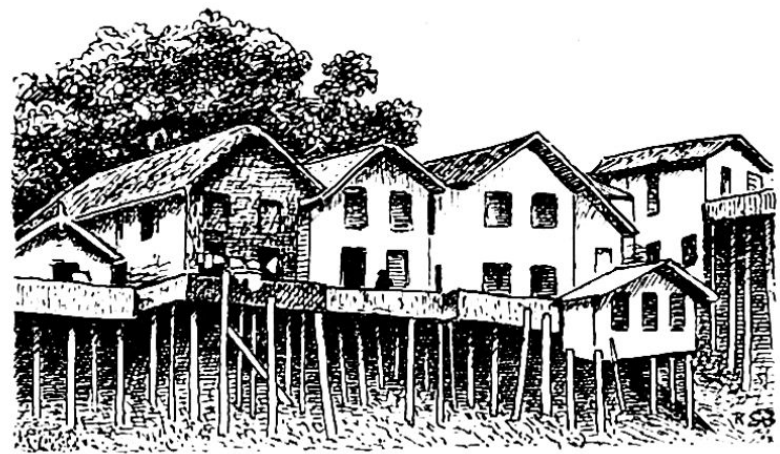

Pfahlbauten im Amazonasgebiet noa, genannt «Montaria», für 2 bis 3 Personen ist etwa $5 \mathrm{~m}$ lang, ausgehöhlt aus einem weichholzigen Baumstamm, zuweilen an den Seiten überhöht durch Bretter. Boote von 6 bis $8 \mathrm{~m}$ Länge heißen "Igarité» und haben hinten ein niederes Dach aus Rundstäben und Palmblättern. «Batalao» heißt das Schiff von 8 bis $10 \mathrm{~m}$ Länge, das auf der hintern Hälfte einMiniaturhaus trägt. Diese Schiffe werden gewöhnlich nicht gerudert, sondern folgen angehängt den motorbetriebenen Chatas. Der Besitzer des Motorschiffes bezieht für den Schlepperdienst Bargeld oder Waren.

In Manaos fallen weiter Gebäude auf, die zur Zeit des Wohlstandes errichtet wurden, so das Theater in fast maurischem Stil, das Instituto Historico e Geografico do Amazonas und viele Häuser von Begüterten. Diese sind dem heißen Klima angepaßt, haben weite Corridore und Veranden, große, hohe Zimmer, Gartenhöfe mit mächtigen Schattenbäumen. Die Häuserfronten beleben vielfach farbige Fliesen. In starkem Kontrast dazu stehen die armseligen Wohnstätten der Eingeborenen in den Außenquartieren, vorwiegend am Ufer des Igarapés. Die Wände der Hütten bestehen aus Brettern oder Rundstäben, das Dach bedeckt mit Sapégras oder Palmblättern, der Fußboden ist festgestampfte Erde. Zuweilen steht die Hütte direkt über dem Wasser auf Pfählen, mit dem Fußboden aus Holz außer Reichweite des Hochwassers, oder aufliegend auf dicken Baumstämmen, welche schwimmend die Niveauveränderungen der Flut mitmachen. Die Hütten sind meistens in 2 bis 3 Räume aufgeteilt, worin viele Menschen gedrängt zusammenleben und in Hängematten schlafen. Im gleichen Raum vollziehen sich Geburt, Geschlechtsleben und Tod. Hygiene wird kaum beachtet. Das Trinkwasser wird dem Fluß entnommen, die Abfälle werden ihm überlassen. $\mathrm{Zu}$ Lande sind die Urubus (Aasgeier) die Sanitätswächter. Es ist nicht zu verwundern, daß man hier vielen Menschen mit Narben oder Flecken ansteckender Krankheiten am Körper begegnet. Rodrigues dos Santos schreibt, daß ihm der Anblick dieses Elends tiefen Eindruck machte, besonders beim Gedanken an den Luxus, der im reichen Südosten des riesigen Landes herrscht. Als Brasilianer durfte er mit Recht auf diese Mißstände hinweisen. Manaos unterhält an einem Igarapé eine Badeanstalt, verbunden mit Vergnügungsstätten. In den Straßen sieht man Menschen aller Stände und Farben spazieren, gutgekleidete neben halbnackten, zerlumpten, in selbstverständlicher Toleranz. Es sind viel mehr Frauen als Männer vorhanden, weil von diesen viele auswärts beschäftigt sind, im Wald, auf der Jagd oder zur Gewinnung des Kautschuks oder in der Industrie. Die Handelsfirmen der Stadt kaufen die Ernten, die aus Kautschuk, Nüssen und Fellen bestehen, den Caboclos ab und liefern ihnen dagegen Stoffe, Werkzeuge, Salz und Petroleum.

Rodrigues dos Santos bereiste den Rio Negro an Bord des Motorschiffes «Joao» von Manaos bis zum Städtchen Tapuruquara, rund $700 \mathrm{~km}$ flußaufwärts. Das Schiff landete an größeren Orten, um Passagiere und Waren auszutauschen, hielt sich während der Fahrt möglichst in Ufernähe, längs der grünen Mauer des prachtvollen üppigen Urwaldes, der tagsüber still ist, aus dem aber nach farbensprühendem Sonnen- 
untergang Geräusche und Stimmen von Tieren aller Art tönen. In den Städten trifft man Menschen verschiedener Rassen, seltener reinblütige Indianer, in geringer $\mathrm{Zahl}$ auch reinblütige Weiße oder Neger, überwiegend jedoch Mischlinge aller drei Hauptrassen, die ethnographisch schwer einzureihen sind.

Der Fisch und das Mandiocamehl bilden die Ernährungsbasis des Caboclos. Honig, Früchte und Getreide kommen in zweiter Linie. Gemüse sind nicht begehrt, sogar der Palmkohl kommt selten auf den Tisch. Mangelkrankheiten sind daher häufig. Die ergiebigste Einnahmequelle des Caboclos ist der Kautschuk. Im Urwald stehen die Bäume nicht in homogenen Beständen beisammen, sondern in buntem Gemisch. Der Caboclo erspäht die Standorte der Gummibäume (Seringueira) und haut mit seinem Buschmesser durch das dichte Unterholz Verbindungspisten von Baum zu Baum. Die Rinde des Gummibaumes wird nach dem Fischgratmuster angeritzt, d. h. zu einer senkrechten Sammelrinne laufen beidseitig schrägabwärts viele Rinnen; unten an der Zentralrinne ist ein Sammelbecher befestigt, in welchen der Saft (Latex) des angeritzten Baumes fließt. Etwa bei jedem dritten Baum ist ein Sammeleimer, in welchen die vollen Becher entleert werden. Die Sammelarbeit verrichtet der Caboclo am frühen Morgen. Nach dem Frühstück geht er an die dritte Arbeit, ans Räuchern. Die Räucherei liegt am Waldrand. Unter einem Dach aus Strohgeflecht, das zwischen zwei Bäumen schräg zum Boden reicht, steht ein primitiver Ofen aus Lehmerde mit Rauchkanälen. Der Rauch wird durch Verbrennen von Abfällen bestimmter Pflanzen erzeugt. Der Caboclo legt nun ein Rundholz auf zwei Gabeln über den Ofen. Auf das Rundholz ist in dessen Mitte etwas Kautschukband als Kern gewickelt. Dann schüttet der Mann (Seringueiro) sukzessive bei drehender Bewegung frischen Latex auf. Dieser gerinnt im Rauch. Hat die Kugel etwa den Durchmesser von $40 \mathrm{~cm}$ erreicht, nimmt sie der Seringueiro vom Ofen und brennt mit einem heißen Eisen seine Marke darauf. Er räuchert täglich etwa 8 bis 10 Liter während zweier Stunden, mithin 70 bis $80 \mathrm{~kg}$ in einer Woche. Der Kautschukballen verliert durch Verdunstung etwas von seinem Gewicht und verändert gleichzeitig seine Farbe von helleuchtendem Weiß zu Dunkelbraun. Neben dem Latex vom Baumstamm gewinnt der Caboclo vom gleichen Baum an den obersten Zweigen einen etwas anders gearteten, fast geruchlosen Saft, der nach besonderer Behandlung zur Herstellung von Peitschen, Isolatoren und für andere industrielle $Z$ wecke Verwendung findet.

Wichtig für den Caboclo ist auch der Fang des Pirarucu, eines fetten Großfisches, dessen Fleisch sehr geschätzt wird. Er wird von der Canoa aus mittelst der Harpune gefangen, wobei die Jäger meistens zu zweit am Morgen ausfahren. Der Pirarucu verrät sein Dasein durch kleine Luftblasen, die er durch das dunkle Wasser aufsteigen läßt. Sobald der dunkle Rücken des Tieres an der Oberfläche erscheint, saust die Harpune nach ihm. Nach langem Kampf gelingt es den Fisch zu überwältigen und an Bord zu ziehen. Er kann $2 \mathrm{~m}$ lang werden, über $100 \mathrm{~kg}$ wiegen und bis 40 Junge haben. Das Fleisch wird eingesalzen und getrocknet. Zuweilen wird beim Fang auch Dynamit gebraucht. Bei dem Raubbau ist die Gefahr des Verschwindens dieser Tierspezies groß. Eine weitere Einnahmequelle bildet für den Caboclo der Fang der Riesenschildkröte (Podocnemys expansa), deren Fleisch eine Volksnahrung und deren Deckschale ein Handelsartikel ist. Der Caboclo, ausgestattet mit von seinen indianischen Vorfahren ererbten Instinkten für erfolgreiche Jagd, schleicht diesem wehrlosen Tier nach. Er weiß, daß im September, mit Beginn der Trockenzeit, die Wasser sinken, große Sandflächen an den Ufern trocken werden und damit die Zeit beginnt, da die Schildkröten hier auf dem heißen Sand ihre Eier ablegen. Die weiblichen Tiere sammeln sich an tiefen Stellen im Flusse, scheinen mit erhobenen Köpfen auf ein Signal zu lauschen und kriechen dann plötzlich in großen Scharen auf den trockenen Sand, halten dort an und spähen aufmerksam umher. Die kürzere, aber höhere männliche Schildkröte steigt aus dem Wasser, ritzt mit dem Schwanz eine kreisförmige 

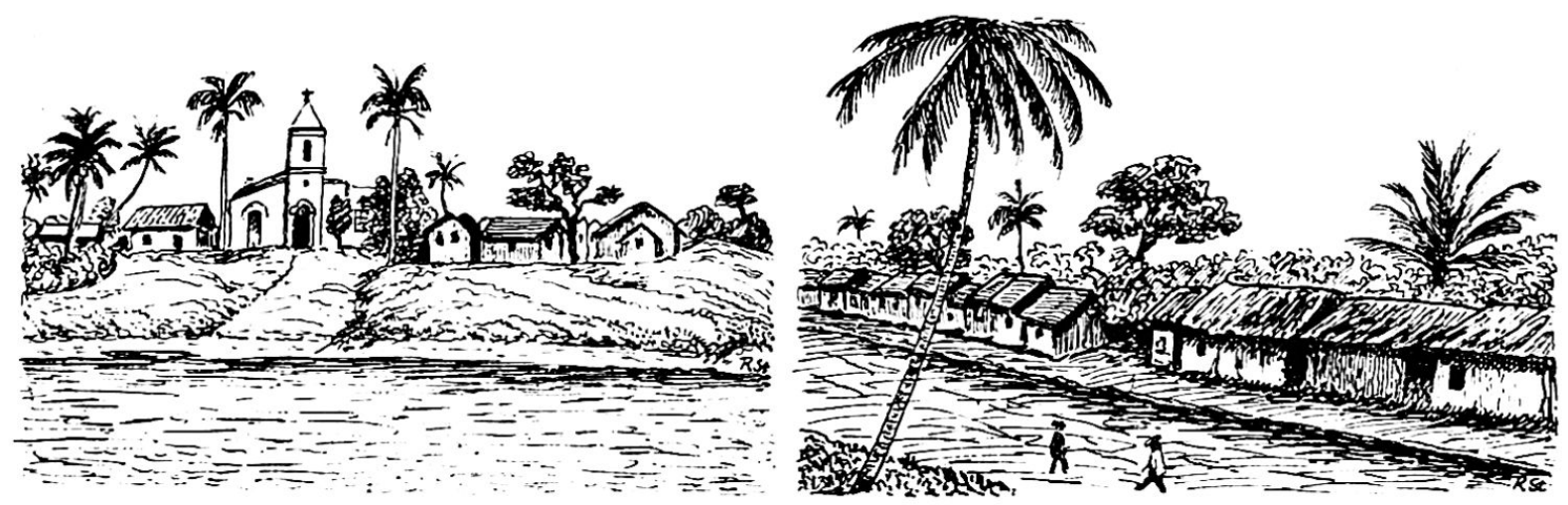

Caoioè, Hauptort im Tal des Rio Negro. Rechts die Hauptstraße

Rinne in den Sand, damit gleichsam den Bezirk abgrenzend, innerhalb welchem nun die Weibchen aufgeregt sichernd, sich einen Platz zur Eierablage aussuchen. Mit den Vorderfüßen schaufeln sie eine Grube von 25 bis $40 \mathrm{~cm}$ Tiefe aus und legen 100 bis 150 Eier hinein. Diese sind rund, etwa von der Größe von Pingpongkugeln, und die Schale ist weich. Bei der Eiablage halten die Tiere ihren Rückenschild schräg nach rückwärts geneigt, die Hinterfüße gegen die Grubenwand gestützt, die Vorderfüße über den Vorderrand der Grube ausgebreitet. Nach der Eiablage decken die Tiere die Eier mit Sand zu, drücken diesen mit ihrer flachen Brust fest an, ebnen und reinigen die Umgebung sorgfältig, um den Ort der Grube unauffällig zu machen und kriechen zum Wasser zurück. Durch die Sonnenhitze werden die Eier ausgebrütet, nach 40 Tagen durchbrechen die Jungen die Schalen und kriechen instinktiv zum Wasser. Sie sind nur etwa 2-3 cm lang. Diese hier beschriebenen Vorgänge verlaufen aber leider nicht so harmlos. Schon zu Anfang tritt der schlimmste Feind der Schildkröte, der Mensch in Aktion. Er lauert im Busch verborgen auf den Moment, da die Schildkröte mit der Eiablage fertig ist, stürzt sich behende auf die Tiere und versucht möglichst viele dieser harmlosen Geschöpfe auf den Rücken zu legen, wodurch sie wehrlos werden, schnürt mit dünnen Lianen Beine und Schale zusammen und trägt sie zur Canoa In der Nähe seiner Hütte ist ein Teich vom Fluß durch eine Pallisade abgetrennt, wohin die Beute gebracht wird. Ein Teil davon dient dem Caboclo und seiner Familie als Nahrung, der Überschuß wird auf den Markt gebracht. Auch von den Eiern eignet sich der Jäger so viel wie möglich an. Für die glücklich aus verborgen gebliebenen Eiern auskriechenden jungen Schildkrötchen ist der kurze Weg zum Wasser gefahrvoll, denn viele Reiher und andere Vögel haben auf diesen Moment gewartet und füllen ihre Kröpfe mit den Kleintieren. Erreichen einige endlich das Wasser, schießen neue Feinde auf sie los, Fische und andere Wassertiere. Nur ein kleiner Prozentsatz bleibt am Leben, so daß die Möglichkeit besteht, daß diese interessante Tierart allmählich gänzlich ausgerottet wird. Ein anderes Tier des Amazonasgebietes ist der Peixe-boi (Seekuh), ein Säugetier von 3 bis $5 \mathrm{~m}$ Länge, das auch mit der Harpune gejagt wird.

Bei der Fahrt mit der Canoa kann man zuweilen an den Uferwänden unter der Wasserlinie dunkle Löcher beobachten. Es sind die Eingänge von Fluchtröhren, die landeinwärts auf Waldboden ausmünden und den Wasserschweinen Paca und Capivara auf der Flucht vor Feinden Schutz bieten. Das Fleisch dieser Jagdtiere ist nicht sehr gesucht, weil es etwas tranig schmeckt.

Der Caboclo treibt nur wenig Ackerbau auf Böden, auf welche die Flut der Regenzeit Düngstoffe heran bringt. Er pflanzt Mandioca, etwas Tabak für Eigengebrauch und Zuckerrohr, in neuerer Zeit auch Jute. Heimindustrie treibt der Caboclo nicht. Neben den wenigen Ackerbauprodukten bringt er nur Überschüsse der Jagd auf den Markt, ferner als Hauptprodukt die Kautschukkugeln, und etwa Fische, Häute und 
Nüsse. Die Hauptmasse des Volkes am Amazonas ist schlecht genährt und analphabetisch. Die Kinder kennen kaum etwas anderes als Wasser, Wald und Wolken am Himmel. Auf dem $\mathrm{km}^{2}$ leben nur 0,4 Bewohner. Wegen den enormen Distanzen ist die Errichtung von Spitälern und Schulen sehr erschwert.

\section{AU BORD DE L'AMAZONE ET DU RIO NEGRO}

En 1957, le Brésilien Rubens Rodriguez dos Santos fit un voyage d'exploration et d'information dans la région de l'Amazone moyen et du Rio Negro. Les deux grands fleuves se ramifient, le long de leurs rives, en de petits canaux latéraux, que l'on nomme «Igarapés». C'est là que les indigènes ont éiu domicile. Ils édifient leurs simples huttes à l'aide de bois et de palmes, sur des hauteurs sèches ou sur des pilotis, à l'abri des hautes eaux. Ils naviguent sur l'Igarapé avec des canoes de différentes grosseurs. Ils équipent l'arrière des plus grandes embarcations d'un toit rond fait de palmes ou même d'une petite hutte. Les Caboclos chassent à l'harpon un gros poisson, le Pirarucu, ou le crocodile. Ils abattent les tortues géantes quand elles rampent sur le sable brûlant pour aller pondre leurs oeufs. La sève de l'arbre à caoutchouc est une bonne source de revenus. Ce liquide laiteux, appelé latex, est épaissi audessus d'un feu dégageant une forte fumée, roulé en boule et, sous cette forme, lancé sur le marché. La capitale régionale, Manaos, connut son apogée au début de ce siècle, lorsque, avec l'apparition de l'automobile, le caoutchouc naturel, provenant d'un arbre de la jungle nommé Maniçoba, fut très recherché. Quand sa demande diminua fortement sous l'effet de la concurrence des plantations de l'Asie sud-orientale, Manaos tomba en décadence. La population est, dans sa plus grande partie, composée de métis. Les gens de pure race sont rares: on vit sous un régime de complète tolérence, paisiblement quoique chétivement.

\section{FAHRT DURCH DIE BRETAGNE}

\section{HANS FrüH}

Vom 16. bis 28. Juli 1958 führte der Verein schweizerischer Geographielehrer eine Studienreise in die Bretagne durch. Die wissenschaftliche Leitung hatte Prof. Dr. H. AnNAheim, Basel, die technische Schulinspektor Dr. H. Liechti, Porrentruy. Jeder Teilnehmer erhielt vor der Exkursion ein vom wissenschaftlichen Leiter verfaßtes Itinerar zur Vorbereitung. Dieses mit Karten und Tabellen versehene vorzügliche Programm leistete sehr gute Dienste. Die Reiseroute umfaßte folgende Tagesetappen: 1. Basel-Auxerre, 2. Auxerre-Tours, 3. Tours -Nantes, 4. Nantes-Vannes, 5. Vannes-Quimper, 6. Quimper-Halbinsel Cornouaille-Quimper, 7. Quimper-Brest, 8. Brest-Trébeurden, 9. Trébeurden-St. Malo, 1o. St. Malo-Alençon, 11. Alençon-Troyes, 12. Troyes-Basel. Die Strecke Basel-Mulhouse wurde per Bahn, die übrige mit einem Autocar gefahren.

Durch die verbreiteten pleistozänen Lößlehme und pliozänen Schotter des Sundgaus gelangten wir nach Belfort mit seinen auf Malmspornen liegenden Fortifikationen. Über die nach Süden einfallenden Sedimenttafeln der südlichen Vogesenausläufer, unter denen die Sargberge der stark bewaldeten Buntsandsteinplatten erkennbar waren, kamen wir ins kleine Steinkohlenbecken von Ronchamp. Der dortige Kohlenbergbau ist seit 1957 eingestellt. Nach Lure, im Zentrum einer von Keuperhügeln umgebenen Ausräumungslandschaft gelegen, durchfuhren wir Vesoul, um bald in den Bereich des Rhône-Saônegrabens einzubiegen. Der Wechsel im geologischen Untergrund, bedingt durch viele Verwerfungen, zeigte sich auch in der landwirtschaftlichen Nutzung. Fast reine Getreidebaulandschaften wechselten mit Gebieten vorherrschenden Wieslandes. Senffelder kündeten die Nähe Dijons an. Viele schreiend gelbe und rote Reklamewände mit Plakaten der Senf- und Essigfabriken aus der burgundischen Hauptstadt besorgten dies aber noch eindrücklicher. Westlich Dijon wurde der Canal de Bourgogne überquert und bei Sombernon die Wasserscheide zum Seinegebiet erklommen. Auf der Höhe liegen vier große Seen zur Wasserhaltung des Canal dc Bourgogne. Bedingt durch die relativ großen Höhenunterschiede in den Ausiäufern des Plateau de Langres, die der Kanal quert, sind viele Schleusen notwendig und damit ist bei entsprechendem Verkehr der Wasserverbrauch groß. Dieser Verkehr scheint aber heute sehr bescheiden zu sein, jedenfalls erblickten wir auf der mehrere Kilometer langen Strecke, auf der wir dem Kanal folgten, nicht ein einziges Schiff. Westlich Sombernon, auf der Fahrt in Richtung Avallon, passierten wir die nördlichsten Ausläufer des Morvan, diesem nördlichsten Massiv im variskischen Streichen des Zentralplateau. Während in den Kalkgebieten eindeutig die Dorfsiedlung dominiert, ist im Kristallin des Morvan der Einzelhof die. vorherrschende Siedlungsform. Diese kupierten Hochflächen beleben bereits Anklänge einer 